\title{
Comparison of Vector Velocity Imaging using Directional Beamforming and Transverse Oscillation for a Convex Array Transducer
}

Jensen, Jørgen Arendt

Published in:

Proceedings of the SPIE - Progress in Biomedical Optics and Imaging

Link to article, DOI:

$10.1117 / 12.2043701$

Publication date:

2014

Document Version

Early version, also known as pre-print

Link back to DTU Orbit

Citation (APA):

Jensen, J. A. (2014). Comparison of Vector Velocity Imaging using Directional Beamforming and Transverse Oscillation for a Convex Array Transducer. In Proceedings of the SPIE - Progress in Biomedical Optics and Imaging (Vol. 9040). [904012] SPIE - International Society for Optical Engineering. https://doi.org/10.1117/12.2043701

\section{General rights}

Copyright and moral rights for the publications made accessible in the public portal are retained by the authors and/or other copyright owners and it is a condition of accessing publications that users recognise and abide by the legal requirements associated with these rights.

- Users may download and print one copy of any publication from the public portal for the purpose of private study or research.

- You may not further distribute the material or use it for any profit-making activity or commercial gain

- You may freely distribute the URL identifying the publication in the public portal 
Paper presented at the SPIE Medical Imaging conference, San Diego, USA, 2014:

\section{Comparison of Vector Velocity Imaging using Directional Beamforming and Transverse Oscillation for a Convex Array Transducer}

Jørgen Arendt Jensen

Center for Fast Ultrasound Imaging,

Biomedical Engineering group, Department of Electrical Engineering, Bldg. 349,

Technical University of Denmark, DK-2800 Kgs. Lyngby, Denmark 


\title{
Comparison of Vector Velocity Imaging using Directional Beamforming and Transverse Oscillation for a Convex Array Transducer
}

\author{
Jørgen Arendt Jensen \\ Center for Fast Ultrasound Imaging, Department of Electrical Engineering, \\ Technical University of Denmark, DK-2800 Lyngby, Denmark
}

\begin{abstract}
Vector velocity imaging can reveal both the magnitude and direction of the blood velocity. Several techniques have been suggested for estimating the velocity, and this paper compares the performance for directional beamforming and transverse oscillation (TO) vector flow imaging (VFI). Data have been acquired using the SARUS experimental ultrasound scanner connected to a BK 8820e (BK Medical, Herlev, Denmark) convex array probe with 192 active elements. A duplex sequence with 129 B-mode emissions interleaved with 129 flow emissions has been made. The flow was generated in a recirculating flow rig with a stationary, laminar flow, and the volume flow was measured by a MAG 3000 (Danfos, Sønderbog, Denmark) magnetic flow meter for reference. Data were beamformed with an optimized transverse oscillation scheme for the TO VFI, and standard fourth-order estimators were employed for the velocity estimation. Directional RF lines were beamformed along the flow direction and cross-correlation employed to estimate the velocity magnitude. The velocities were determined for beam-to-flow angles of 60,75 and 90 degrees. Using 32 emissions the standard deviation relative to the peak velocity for TO estimation was $7.0 \%$ at a beam-to-flow angle of $75^{\circ}$. This was $3.8 \%$ for directional beamforming and at $60^{\circ}$ it was $2.2 \%$. The general improvement, however, comes at an increase by a factor of roughly 11 in the number of calculations for the directional beamformation compared to the TO method.
\end{abstract}

\section{INTRODUCTION}

Vector velocity estimation is now being introduced in clinical use for studying the hemodynamics of the human body ${ }^{1}$ and for visualizing complex flow phenomena. ${ }^{2}$ The commercially available scanners are based on the transverse oscillation technique ${ }^{3-5}$ that introduces a laterally oscillating field to make it sensitive to the transverse velocity component. This is a very computationally efficient method, but it relies on a complicated transverse oscillating ultrasound field. Several other techniques have been introduced which might improve on the estimation accuracy. These include speckle tracking ${ }^{6}$ and using spectral broadening. ${ }^{7}$ Bonnefous ${ }^{8}$ suggested using beamforming transverse to the ultrasound field and cross-correlation to determine the lateral motion. Another approach is to use beamforming along the flow direction as suggested by Jensen and Bjerngaard. ${ }^{9,10}$ None of these methods have, however, reached a commercial implementation. A review of the early history of vector Doppler has been published by Dunmire and Beach. ${ }^{11}$

Currently the transverse oscillation (TO) approach is implemented on the commercial BK Medical scanners using a linear array probe. This should be expanded to convex array probes for increasing the penetration depth and field of view. It is, however, interesting whether this is the best approach for vector velocity estimation. In this paper the optimized TO method ${ }^{12}$ using a convex array probe is therefore compared to the directional velocity estimation method. Data acquired by the SARUS experimental scanner have be used and processed off-line to reveal the performance for a stationary, parabolic velocity profile.

\section{VECTOR VELOCITY ESTIMATION METHODS}

The TO method estimates the velocity vector by introducing a lateral oscillation in the ultrasound field. This is made by sending out a weakly focused field and then beamforming three signals in parallel during receive processing: a traditional beam for axial velocity estimation, and a complex set of lateral oscillating beams. The latter two beams are made using a receive apodization with two peaks, which thereby generates the lateral 


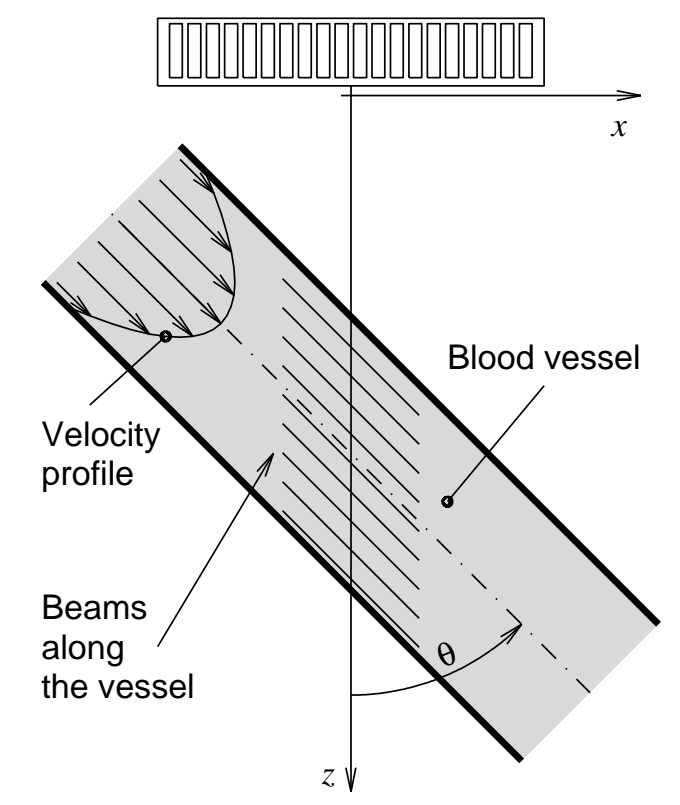

Figure 1. The directional signals shown inside the vessel are focused along the flow vector direction $\left(\right.$ from $\left.{ }^{9}\right)$.

oscillation. ${ }^{3}$ They are focused a quarter lateral wavelength apart to form a spatial in-phase and a quadrature signal $r_{s q}(i)$ suitable for velocity estimation, where $i$ is the emission number.

The temporal Hilbert transform of the received signal is $r_{s q h}(i)$. Two new signals are then formed from:

$$
\begin{aligned}
& r_{1}(i)=r_{s q}(i)+j r_{s q h}(i) \\
& r_{2}(i)=r_{s q}(i)-j r_{s q h}(i)
\end{aligned}
$$

The estimators are then: ${ }^{5}$

$$
v_{x}=\frac{d_{x}}{2 \pi 2 T_{p r f}} \arctan \left(\frac{\Im\left\{R_{1}(1)\right\} \Re\left\{R_{2}(1)\right\}+\Im\left\{R_{2}(1)\right\} \Re\left\{R_{1}(1)\right\}}{\Re\left\{R_{1}(1)\right\} \Re\left\{R_{2}(1)\right\}-\Im\left\{R_{1}(1)\right\} \Im\left\{R_{2}(1)\right\}}\right)
$$

and

$$
v_{z}=\frac{c}{2 \pi 4 T_{p r f} f_{t}} \arctan \left(\frac{\Im\left\{R_{1}(1)\right\} \Re\left\{R_{2}(1)\right\}-\Im\left\{R_{2}(1)\right\} \Re\left\{R_{1}(1)\right\}}{\Re\left\{R_{1}(1)\right\} \Re\left\{R_{2}(1)\right\}+\Im\left\{R_{1}(1)\right\} \Im\left\{R_{2}(1)\right\}}\right) .
$$

where $R_{1}(1)$ is the complex lag one autocorrelation value for $r_{1}(i)$ and $R_{2}(1)$ is the complex lag one autocorrelation value for $r_{2}(i)$. $\Im$ denotes imaginary part and $\Re$ real part. $T_{p r f}$ is the time between two pulse emissions, $f_{t}$ is the transducer center frequency, $d_{x}$ is the lateral oscillation period, and $c$ is the speed of sound. The estimators resemble the ordinary autocorrelation estimator ${ }^{13}$ but uses fourth order correlations instead of second order. They are therefore efficient to implement compared to cross-correlations methods.

The directional beamforming approach is illustrated in Fig. 1. Here the received channel data are beamformed along the flow direction, and the responses are cross-correlated to find the shift in position, and, thus, velocity of the blood. The advantage of the approach is that there is negligible de-correlation between beamformed signals, and that ensures a precise estimate of the velocity. A standard delay-and-sum beamformation is employed, but the points for beamforming are placed along a line following the flow direction. The signal is beamformed with a distance between samples of $\lambda / N_{d}$, where $\lambda$ is the wavelength and $N_{d}$ is the number of points per wavelength, usually around 10 . The line length is $10 \lambda$ in this paper. Correlating such lines will give a correlation function where the peak value is placed at the displacement between emissions. Dividing by the time between emission, thus, yields the velocity magnitude. ${ }^{9}$ 


\section{CALCULATION LOAD}

The number of calculations for the two methods are very different, as the TO method only uses two complex samples at each depth, whereas the directional cross-correlation approach uses a full beamformed line. It is therefore considerably more computationally intensive to implement.

For the directional beamformation method the number of calculations per second is

$$
N_{d}=\left(N_{l}^{2}+N_{l}\right) \frac{f_{s}}{N_{s}} F_{f},
$$

where $N_{l}$ is the number of samples in a directional line, $N_{s}$ is the number of RF samples between velocity estimates, $f_{s}$ is the sampling frequency and $F_{f}$ is the fraction of time spend on velocity estimation. The first term $N_{l}^{2}$ is the cross-correlation estimator, the second is the summing of the correlation functions, and the last term is the number of correlation functions per second.

For the TO estimator the number of calculations is

$$
N_{T O}=\left(4 \cdot 28 N_{s}\right) \frac{f_{s}}{N_{s}} F_{f},
$$

where 28 is the number of complex operations needed to calculate the estimator for one set of RF input samples and 4 calculations have to be made for each complex operation. The ratio between the two methods is:

$$
R_{c}=\frac{\left(N_{l}^{2}+N_{l}\right) f_{s} / N_{s} F_{f}}{\left(4 \cdot 28 N_{s}\right) f_{s} / N_{s} F_{f}}=\frac{\left(N_{l}^{2}+N_{l}\right)}{\left(4 \cdot 28 N_{s}\right)} .
$$

Typical values are $N_{l}=100, N_{s}=8$, which gives $R_{c}=11.3$. The TO estimator is, thus, 11 times more efficient in terms of calculations. It demands around 980 Mflops, when half the time is efficiently used for flow estimation and sampling at $17.5 \mathrm{MHz}$ with half a pulse length between velocity estimates. For the directional approach it demands around 11 Gflops, which is still within the reach of modern GPU cards.

It should also be noted that considerably more beamforming takes place for the directional approach. Three parallel beams have to be made along with a temporal Hilbert transform for the TO, whereas $N_{l}$ beamforming operations have to take place for each RF sample received for the directional approach. It has also been assumed that the angle determination has been made before the directional signals are beamformed. This also decreases the number of calculations significantly. ${ }^{14}$

\section{MEASUREMENT SETUP}

The SARUS experimental ultrasound scanner has been used for acquiring data for both approaches. ${ }^{15}$ The scanner can acquire full RF data where the signal from each transducer element is sampled. The data are sampled at $70 \mathrm{MHz}$ and then decimated by a factor of 4 for an effective sampling frequency of $17.5 \mathrm{MHz}$ with 12-bits precision. A $3 \mathrm{MHz}$ BK 8820e (BK Medical, Herlev, Denmark) convex array probe was used in a duplex mode setup. A 129 line B-mode image was interleaved with 129 flow emissions in one direction. The sequence was made continuous, so that data can be used across image boundaries. The probe has 192 elements, and the focus in transmit was at $40 \mathrm{~mm}$ for B-mode imaging and at $105 \mathrm{~mm}$ for the flow emissions. A four cycle pulse was used for the flow acquisition, and the same data were used both for directional beamforming and TO imaging to maintain the same measurement conditions in terms of focusing and signal-to-noise ratio. Measurements were conducted on a re-circulating flow rig with a stationary, parabolic flow profile. The tube had a radius of $6 \mathrm{~mm}$, and the $1.2 \mathrm{~m}$ entrance length to the measurement site ensured a fully developed parabolic velocity profile. A MAG 3000 (Danfoss, Sønderborg, Denmark) magnetic flow meter was used as a reference. Ten frames where measured for each of the beam-to-low angles of 60, 75 and 90 degrees, and all data were stored for off-line processing in Matlab under Linux. Each measurement thereby yielded 1290 pulse-echo flow lines with 750 Mbytes of data. 

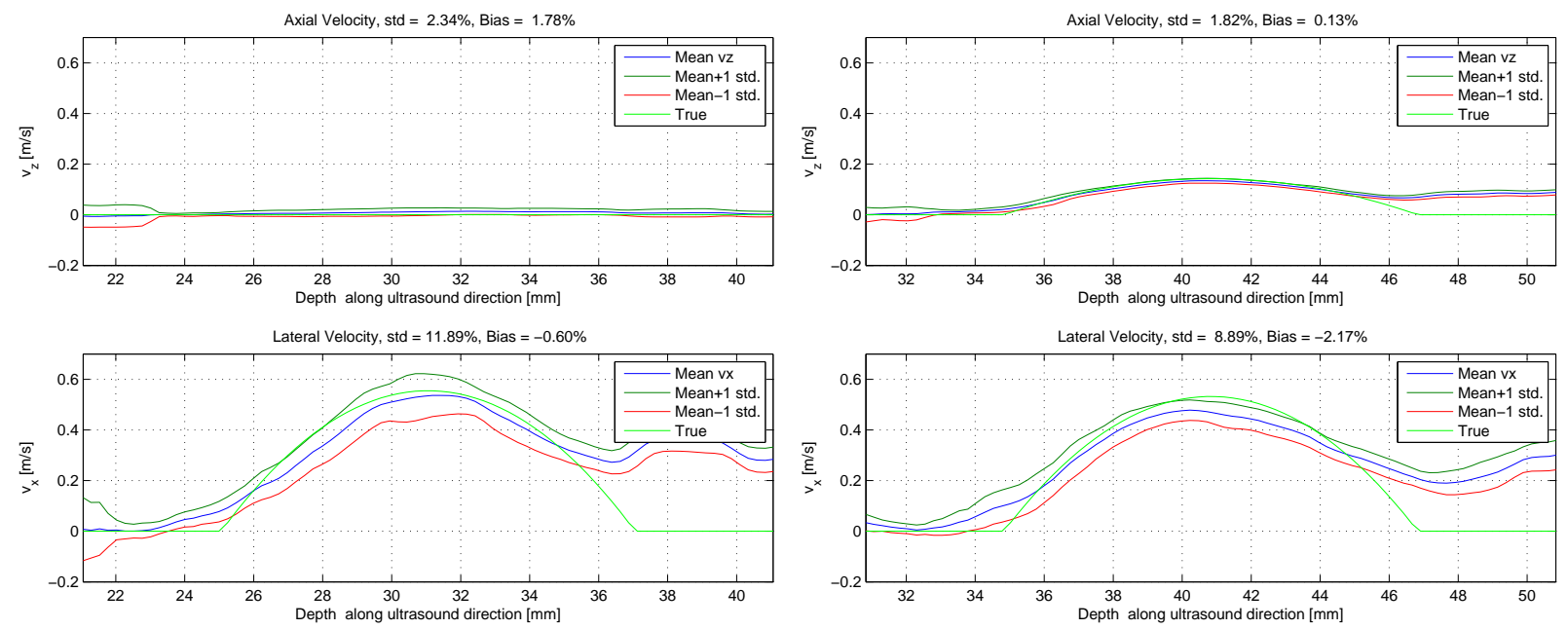

Figure 2. Mean measured velocity profiles for the TO method at a $90^{\circ}$ and $75^{\circ}$ flow angles for the axial (top) and lateral (bottom) velocity components. The green curve indicates the true velocity profile.

\section{RESULTS}

The mean estimated velocity profiles for the TO method are shown in Fig. 2. Sixteen emissions have been employed for each velocity estimate, and the graphs show the results for 80 estimates. The mean profile \pm one standard deviation compared to the true profile are shown for both the axial and lateral velocity components. The left graphs are for a beam-to-flow angle of $90^{\circ}$ and the right for $75^{\circ}$. A standard deviation (STD) of $11.9 \%$ relative to the peak velocity in the vessel is obtained at $90^{\circ}$ and $8.9 \%$ at $75^{\circ}$. The bias is moderate at $-0.5 \%$ and $2.1 \%$ averaged over the profile, although a slightly lower peak velocity and a broadening of the profile is seen. It should be noted that the measurements are conducted for a tube surround by water without scattering. There is, thus, no signal on which to perform the usual discrimination between tissue and flow on, and that is why the profile is unrealistically high outside the vessel. This would be remedied by employing a discriminator, which would set velocities outside the vessel to zero.

The corresponding results for the directional beamforming approach are shown in Fig. 3. The relative STD is $6.0 \%$ at $90^{\circ}$ and $2.8 \%$ at $75^{\circ}$, which is a factor of $2-3$ lower than the TO approach. It should, however, be noted that the angle has been predetermined for the directional beamforming, and, thus, the angle determination does not affect the estimation accuracy. The bias is somewhat higher at $-8.6 \%$ and $3.0 \%$, but it is still at an acceptable level. It can probably also be decreased by optimizing the apodization used during the beamformation. Especially close to the transducer it is probably not ideal to use 128 elements during receive processing.

The overall variations in bias and standard deviation as a function of the number of emissions for the TO methods are shown in Fig. 4 for beam-to-flow angles of $90^{\circ}, 75^{\circ}$, and $60^{\circ}$. The performance at two depths are shown on the top figures at $90^{\circ}$. At a depth of $31 \mathrm{~mm}$ a low bias is obtained and the standard deviation decreases with the number of emissions. It is always higher for the lateral than the axial component. This pattern is also seen at a depth of $50 \mathrm{~mm}$, although there is a consistent bias for the lateral velocity. This indicates that the lateral oscillation period could be optimized.

The lateral velocity estimates have a slightly higher standard deviation at $75^{\circ}$, but the bias is lower for a depth of $40 \mathrm{~mm}$. The same pattern is seen at $60^{\circ}$, and this indicates that an increased axial velocity influences the lateral velocity estimates.

The velocity magnitude for the TO method was calculated and the performance evaluated. This is shown in Fig. 5 and the same overall trend as in Fig. 4 is seen. The same evaluation for the directional velocity estimation is shown in Fig. 6 for the standard deviation (left) and bias (right). Here the highest STD is found at a $90^{\circ}$ beam-to-flow angle where it is most difficult for the correlation estimator to determine the shift. The STD is, however, lower than for the TO method. A clear improvement is seen at $60^{\circ}$ and $75^{\circ}$, which performs significantly 

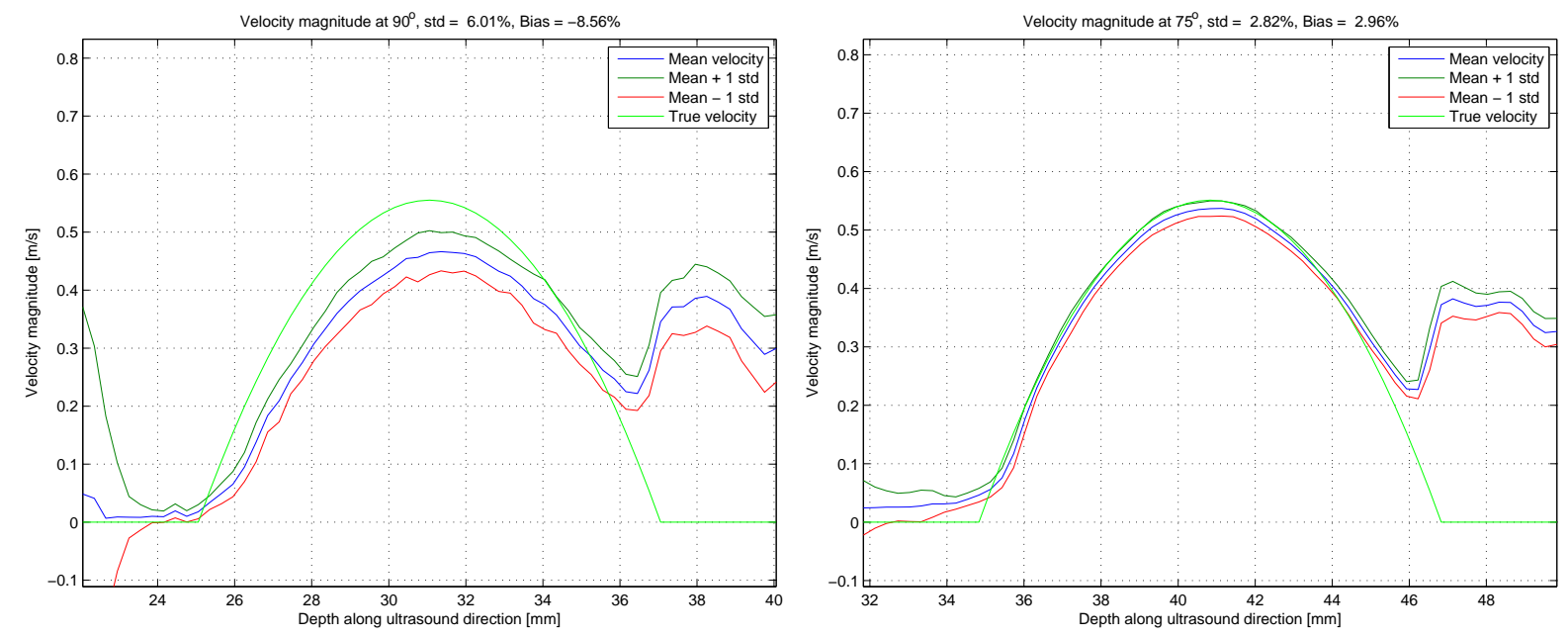

Figure 3. Mean measured velocity profiles for the directional beamforming method at a $90^{\circ}$ and $75^{\circ}$ flow angle for the axial (top) and lateral (bottom) velocity components. The green curve indicates the true velocity profile.

better than the TO approach. The bias is also most affected by the angle at $90^{\circ}$, where a consistently lower estimate of the velocity is seen.

\section{DISCUSSION AND CONCLUSION}

The transverse oscillation and directional beamforming approaches have been compared using the SARUS experimental scanner. Data were measured using a convex array probe on a circulating flow rig with stationary flow. Both methods can reliably find the velocity magnitude. For the TO method 20 emissions have to be used for keeping the standard deviation below $10 \%$ for all depths and angles. Ten emissions are sufficient for the directional lines. The directional beamforming approach can in general reduce the relative standard deviation by roughly a factor of 2 compared to the TO method. The two methods are fairly un-biased for most situation (below 5\%) and can probably be made completely un-biased by further optimizing the receive beamforming either by adjusting the apodization for directional beamforming or by adjusting the lateral wavelength for the TO method.

It should, however, be noted that the improved performance of the directional method comes at a very substantial increase in the amount of calculations. Both the beamformation and velocity estimator are more computationally intensive for the directional method, and it should be further noticed that the beam-to-flow angle has been known prior to beamforming. This can be estimated as described in, ${ }^{16}$ but again with a substantial increase in the number of calculations.

\section{REFERENCES}

[1] P. M. Hansen, M. M. Pedersen, K. L. Hansen, M. B. Nielsen, and J. A. Jensen, "Demonstration of a vector velocity technique," Ultraschall in Med. 32, pp. 213-215, 2011.

[2] K. L. Hansen, J. Udesen, F. Gran, J. A. Jensen, and M. B. Nielsen, "In-vivo examples of complex flow patterns with a fast vector velocity method," Ultraschall in Med. 30, pp. 471-476, 2009.

[3] J. A. Jensen and P. Munk, "A New Method for Estimation of Velocity Vectors," IEEE Trans. Ultrason., Ferroelec., Freq. Contr. 45, pp. 837-851, 1998.

[4] M. E. Anderson, "Multi-dimensional velocity estimation with ultrasound using spatial quadrature," IEEE Trans. Ultrason., Ferroelec., Freq. Contr. 45, pp. 852-861, 1998.

[5] J. A. Jensen, "A New Estimator for Vector Velocity Estimation," IEEE Trans. Ultrason., Ferroelec., Freq. Contr. 48(4), pp. 886-894, 2001. 

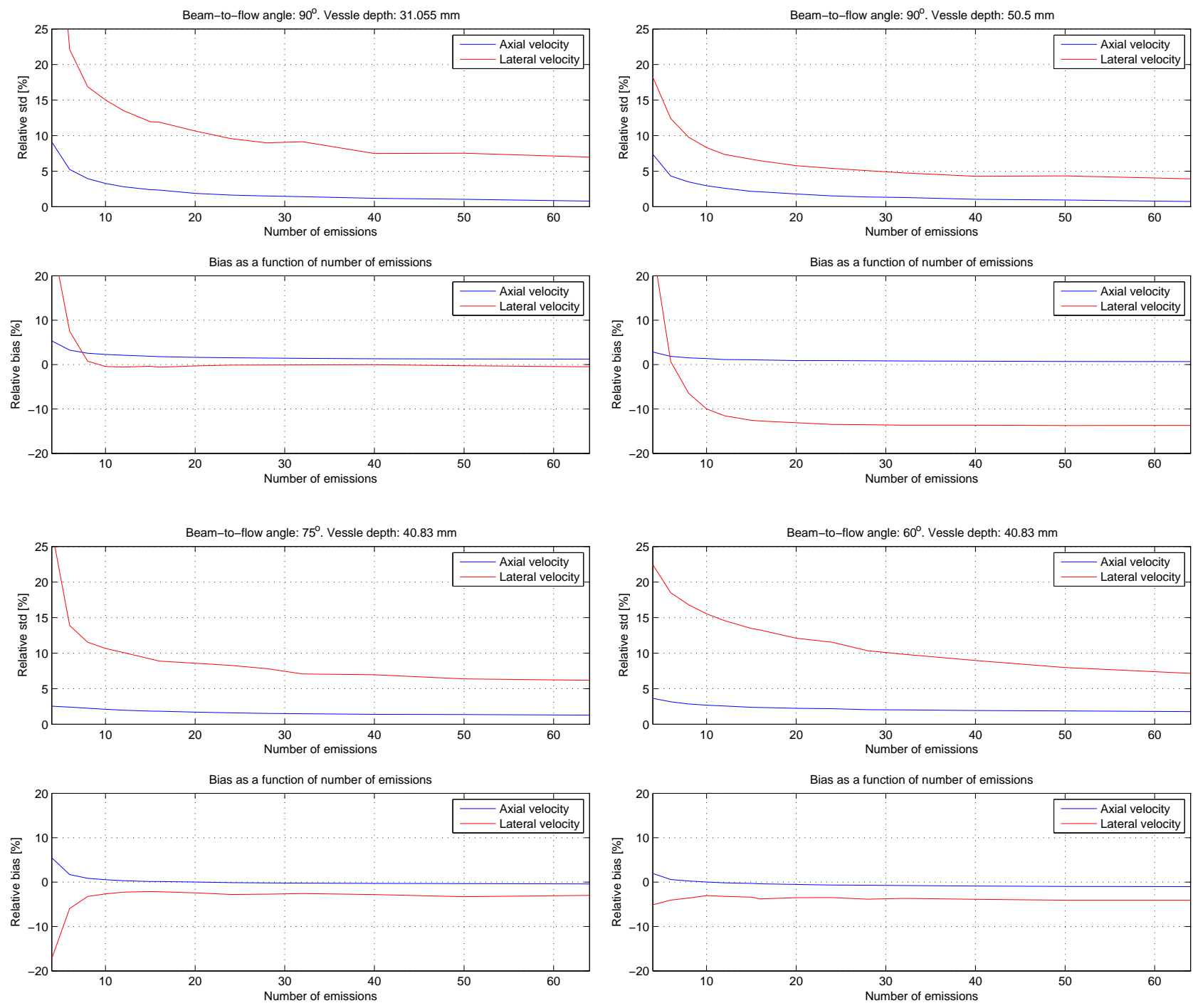

Figure 4. Variation in performance as a function of number of lines for the TO method for both the lateral and axial velocity estimates for a number of beam-to-flow angles and measurement depths. 

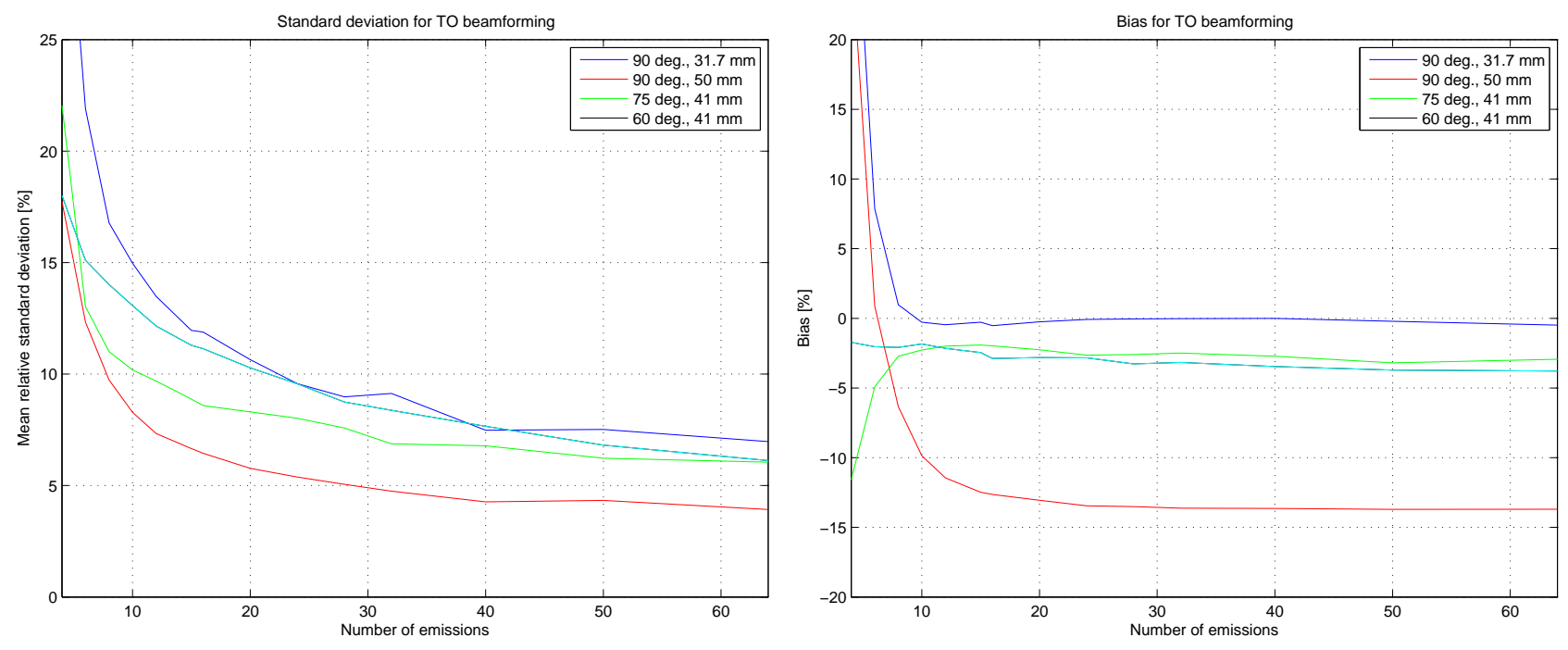

Figure 5. Variation in in performance as a function of the number of lines for the velocity magnitude for the TO approach. The relative standard deviation are shown on the left graphs and the relative bias on the right for the different beam-to-flow angles and depths.
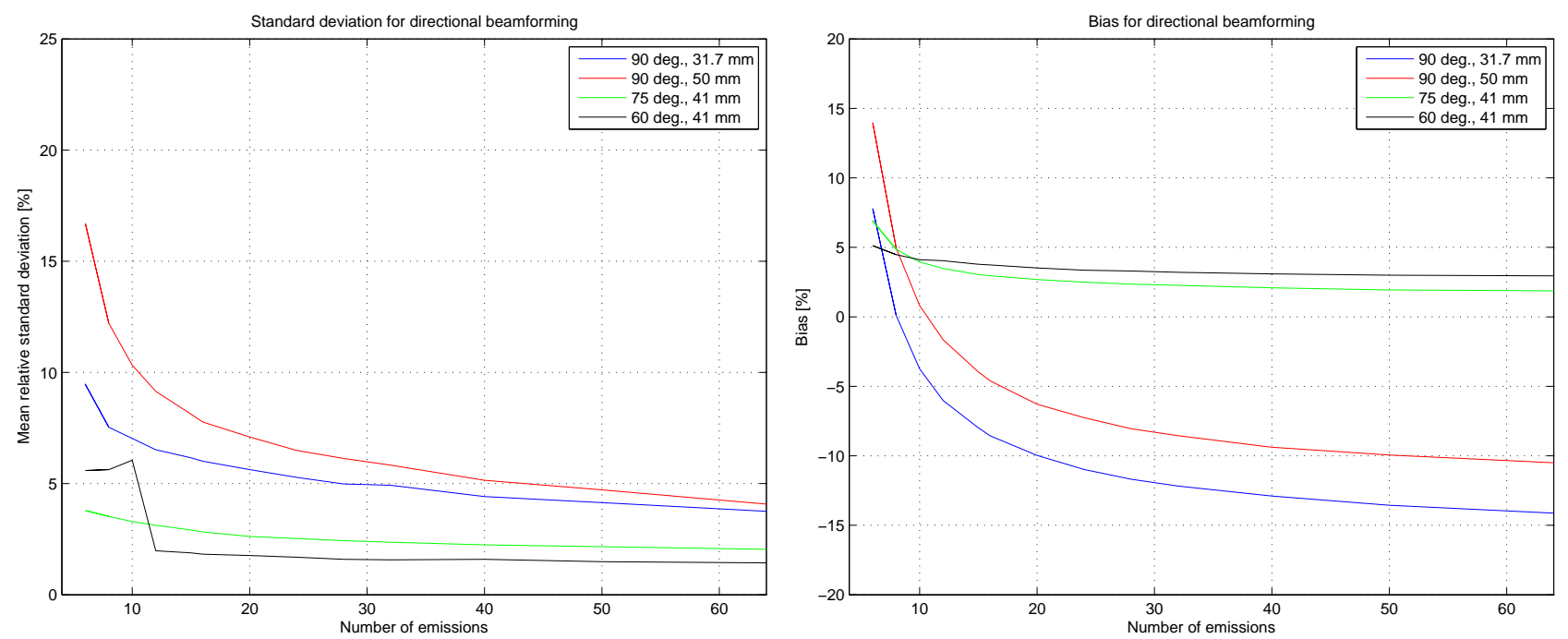

Figure 6. Variation in in performance as a function of the number of lines for the directional beamforming approach. The relative standard deviation are shown on the left graphs and the relative bias on the right for the different beam-to-flow angles and depths. 
[6] G. E. Trahey, J. W. Allison, and O. T. von Ramm, "Angle independent ultrasonic detection of blood flow," IEEE Trans. Biomed. Eng. BME-34, pp. 965-967, 1987.

[7] V. L. Newhouse, D. Censor, T. Vontz, J. A. Cisneros, and B. B. Goldberg, "Ultrasound Doppler probing of flows transverse with respect to beam axis," IEEE Trans. Biomed. Eng. BME-34, pp. 779-788, 1987.

[8] O. Bonnefous, "Measurement of the complete (3D) velocity vector of blood flows," in Proc. IEEE Ultrason. Symp., pp. 795-799, 1988.

[9] J. A. Jensen, "Directional velocity estimation using focusing along the flow direction: I: Theory and simulation," IEEE Trans. Ultrason., Ferroelec., Freq. Contr. 50, pp. 857-872, 2003.

[10] J. A. Jensen and R. Bjerngaard, "Directional velocity estimation using focusing along the flow direction: II: Experimental investigation," IEEE Trans. Ultrason., Ferroelec., Freq. Contr. 50, pp. 873-880, 2003.

[11] B. Dunmire and K. W. Beach, "A Brief History of Vector Doppler," in Proc. SPIE Med. Imag., 4325, pp. 200-214, Feb. 2001.

[12] J. A. Jensen, "Optimization of transverse oscillating fields for vector velocity estimation with convex arrays," in Proc. IEEE Ultrason. Symp., pp. 1753-1756, July 2013.

[13] C. Kasai, K. Namekawa, A. Koyano, and R. Omoto, "Real-Time Two-Dimensional Blood Flow Imaging using an Autocorrelation Technique," IEEE Trans. Son. Ultrason. 32, pp. 458-463, 1985.

[14] J. Kortbek and J. A. Jensen, "Estimation of velocity vector angles using the directional cross-correlation method," IEEE Trans. Ultrason., Ferroelec., Freq. Contr. 53, pp. 2036-2049, 2006.

[15] J. A. Jensen, H. Holten-Lund, R. T. Nilsson, M. Hansen, U. D. Larsen, R. P. Domsten, B. G. Tomov, M. B. Stuart, S. I. Nikolov, M. J. Pihl, Y. Du, J. H. Rasmussen, and M. F. Rasmussen, "SARUS: A synthetic aperture real-time ultrasound system," IEEE Trans. Ultrason., Ferroelec., Freq. Contr. 60(9), pp. 1838-1852, 2013.

[16] J. A. Jensen and N. Oddershede, "Estimation of velocity vectors in synthetic aperture ultrasound imaging.," IEEE Trans. Ultrason., Ferroelec., Freq. Contr. 25, pp. 1637-1644, 2006. 